\title{
El análisis de las fortalezas psicológicas en la adolescencia: Más allá de los modelos de vulnerabilidad
}

\author{
Montse Giménez ${ }^{1 *}$, Carmelo Vázquez ${ }^{2}$, and Gonzalo Hervás ${ }^{2}$ \\ ${ }^{1}$ Escuela Universitaria Cardenal Cisneros (Alcalá de Henares, Madrid) \\ ${ }^{2}$ Facultad de Psicología, Universidad Complutense de Madrid \\ (Received May 15, 2010; Accepted September 24, 2010)
}

\begin{abstract}
RESUMEN: Frente a la imagen traumática y conflictiva que se asocia tradicionalmente a la adolescencia, desde el marco de la Psicología Positiva se ofrece una nueva conceptualización que sugiere una visión más optimista y positiva de este periodo de la vida. El estudio de las fortalezas humanas, término descrito por Peterson y Seligman (2004), ofrece un contexto potencialmente útil para conocer los aspectos y cualidades positivas de los más jóvenes y apuesta por un modelo de intervención centrado en las potencialidades frente al déficit. El presente artículo realiza una revisión sobre los resultados encontrados en la literatura que, de modo directo o indirecto, apoyan la existencia y relevancia de cada una de las fortalezas señaladas en el marco descriptivo de estos autores. Finalmente, discutimos las repercusiones que el estudio de las potencialidades puede tener en el desarrollo de programas educativos, de prevención y de intervención.
\end{abstract}

Palabras clave: psicología positiva, fortalezas, desarrollo humano, adolescencia.

The analysis of the psychological strengths in adolescence: Beyond vulnerability models

ABSTRACT: Against a traumatic and troubled image that is traditionally associated with adolescence, Positive Psychology opens a novel conceptual framework offering a more optimistic and positive view of this period of life. The study of 'human strengths', a term suggested by Peterson and Seligman (2004), offers a potentially useful tool to understand the positive aspects and features of the youth. That approach can also provide a model of intervention focusing on individual's potentials rather than on deficits. This paper reviews the results found in the literature that directly or indirectly supports the existence and relevance of each of the strengths identified in the descriptive framework of these authors. Finally, we discuss the implications that the study of human strengths may have in the development of educational, preventive and intervention programs.

Key words: positive psychology, strengths, human development, adolescence.

Aunque el estudio de las capacidades humanas ha sido siempre un objetivo importante de la Psicología y la Educación, en temas tan variados como la creatividad, la inteligencia, el altruismo, o el amor (ver Fernández Ballesteros, 2002), desde mediados del siglo XX, y por razones históricas diversas, estas disciplinas se han centrado más en el análisis de los déficits y las fragilidades humanas que en la exploración y promoción de tales capacidades (Seligman, 2003). 
Desde que en enero del año 2000, la revista American Psychologist publicara un número especial sobre Psicología Positiva (movimiento liderado principalmente por Seligman y Csikzentmihalyi), el interés hacia la promoción del funcionamiento óptimo, y no sólo la eliminación del malestar o la superación de los déficits, ha venido cobrando un papel relevante en la Psicología actual (Vázquez y Hervás, 2009). Dentro de este movimiento, se considera que en el estudio de la naturaleza humana se debe emplear una visión complementaria, que no alternativa, para comprenderla en su plenitud. Frente a una visión muy sesgada hacia lo negativo, este movimiento enfatiza el estudio de las emociones positivas y el bienestar, de las capacidades psicológicas, y de las instituciones positivas (ver Vázquez, 2009).

Si bien todas las etapas evolutivas son determinantes en el desarrollo de una persona adulta, tradicionalmente se ha ido configurando una conceptualización de la adolescencia como un período de la vida especialmente conflictivo y traumático. Esta visión generalizada de padres, educadores y sociedad, constituye el principal obstáculo para establecer relaciones positivas desde las cuales permitir el desarrollo de las potencialidades de los más jóvenes. Frente a esta visión, surgen estudios que apuestan por adoptar un punto de vista diferente: el adolescente, no como fuente de problemas, sino como un valioso recurso en proceso de desarrollo (Arguedas y Jiménez, 2007).

No hay que olvidar que dentro de los objetivos del estudio y análisis de fortalezas iniciado por Peterson y Seligman (2004) se encuentra no sólo el desarrollo de fortalezas sino, casi más importante, el reconocimiento y el pleno aprovechamiento de estas. En ocasiones, tanto personas como instituciones, invierten mucho esfuerzo en cumplir un objetivo, corregir una limitación o potenciar un rasgo positivo. Sin embargo, en ocasiones, es más sencillo y eficaz para alcanzar dicho objetivo, usar la vía del reconocimiento de las fortalezas ya existentes y la puesta en funcionamiento de las mismas en una variedad de ámbitos. Los estudios con población adulta sugieren que el logro de metas con un componente intrínseco importante (y las fortalezas innatas lo son de forma muy marcada), tiene un impacto mucho mayor sobre el bienestar que el logro de aquellas que no tienen dicho componente (Sheldon y Elliot, 1999). Probablemente, en la adolescencia, el reconocimiento de las fortalezas personales puede tener además un papel clave en el asentamiento de la identidad y de la autoestima.

En el presente trabajo haremos una breve revisión sobre el concepto de fortaleza psicológica, término que hace referencia a las características o manifestaciones psicológicas de las virtudes clásicas (Peterson y Seligman, 2004), con un foco en las investigaciones efectuadas en el ámbito de la infancia y la juventud.

\section{FORTALEZAS HUMANAS}

En 1999, un grupo de investigadores entre los que destacan Mihaly Csikszentmihalyi, Ed Diener, Martin Seligman, y principalmente, Christopher Peterson, iniciaron el trabajo de crear una lista de fortalezas humanas que permitiera 
profundizar en el desarrollo de una psicología centrada en los rasgos positivos. Peterson y Seligman continuaron este trabajo, proponiendo una taxonomía final formada por veinticuatro fortalezas humanas (Peterson y Seligman, 2004).

A partir de la revisión de diferentes escritos y manifestaciones filosóficas, teológicas y culturales, descubrieron que en la mayoría de los documentos revisados se valoraban seis virtudes:

1. La sabiduría y el conocimiento: engloba las fortalezas cognitivas relacionadas con la adquisición y el uso del conocimiento.

2. El valor: fortalezas emocionales relacionadas con actuaciones conscientes dirigidas a objetivos encomiables, realizados ante fuertes adversidades y ante la incertidumbre de si podrán ser conseguidos o no.

3. El amor y la humanidad: fortalezas interpersonales que incluyen acercamiento y amistad con otros.

4. La justicia: fortalezas cívicas que persiguen una vida en comunidad saludable.

5. La templanza: fortalezas que protegen contra el exceso y facilitan la expresión apropiada y moderada de los apetitos y necesidades.

6. La espiritualidad y la trascendencia: fortalezas que conectan con el universo más amplio y proporcionan un significado a la vida.

Los autores consideran el concepto de virtud como algo demasiado amplio y abstracto y empiezan a utilizar el concepto de fortaleza para referirse a la manifestación psicológica de la virtud. En otras palabras las fortalezas humanas serían los ingredientes (procesos o mecanismos) psicológicos que definen las virtudes (Peterson y Seligman, 2004). En un segundo momento, identificaron una serie de criterios específicos que determinarían si una fortaleza podría incluirse o no en la clasificación definitiva. En la tabla 1 se recogen los criterios utilizados.

\section{Tabla 1. Criterios para la Selección de las Fortalezas (Peterson y} Seligman, 2004)

- $\quad$ Es necesario que la fortaleza sea ampliamente reconocida y celebrada por las diferentes culturas.

- Una fortaleza debe contribuir al desarrollo de una vida plena, satisfactoria y feliz.

- Aunque las fortalezas pueden conllevar y conllevan consecuencias positivas, cada fortaleza es valorada moralmente en sí misma, aún cuando no es seguida de consecuencias beneficiosas.

- El desarrollo de una fortaleza en una persona no disminuye el desarrollo en otras personas.

- Es necesario que no exista un antónimo de la fortaleza en términos positivos.

- Una fortaleza debe manifestarse en la conducta de un individuo.

- La fortaleza se distingue de otros rasgos positivos en su clasificación y 
no puede ser descompuesta en otras.

- Es necesario que existan diferentes manifestaciones culturales de esa fortaleza, a través de historias, canciones, poemas...

- Es posible encontrar las fortalezas en su forma pura o extrema, es decir, ejemplos de personas virtuosas en alguna fortaleza.

- Cabe esperar la existencia de prodigios con respecto a las fortalezas

- $\quad$ Puede haber gente que no muestre, selectivamente, alguna fortaleza.

- La mayoría de las sociedades ha provisto de instituciones y/o rituales con el objetivo de cultivar fortalezas y virtudes para favorecer su práctica.

A partir de la revisión de los diferentes escritos y después de definir los diferentes criterios, describen 24 fortalezas clasificadas en seis subgrupos (ver tabla 2).

Los autores ya dejaron claro en su texto inicial (Peterson y Seligman, 2004), que la clasificación es tan sólo una propuesta inmersa en un proceso de construcción abierto y flexible y que, como otros modelos de clasificación (como el del DSM), necesita de tiempo, investigación y estudio para su desarrollo y consolidación.

Paralelamente al desarrollo de la clasificación teórica de las fortalezas humanas, Peterson y Seligman, en colaboración con otros autores, han ido diseñando instrumentos de medida que permiten recoger datos en poblaciones muy diversas y conocer no sólo si existen o no esas fortalezas, sino los diferentes grados que pueden presentar.

\section{Tabla 2. Virtudes Humanas y Definición de las Fortalezas Asociadas (Peterson y Seligman, 2004)}

\begin{tabular}{|c|c|c|}
\hline Virtud & Fortaleza humana & Definición \\
\hline \multirow[t]{4}{*}{ Sabiduría } & Creatividad & $\begin{array}{l}\text { Pensar nuevas y productivas formas de conceptualizar y } \\
\text { hacer las cosas; incluye la creatividad artística, pero no se } \\
\text { limita a ésta. }\end{array}$ \\
\hline & Curiosidad/interés & $\begin{array}{l}\text { Interesarse por el mundo, encontrar temas de interés, estar } \\
\text { abierto a la experiencia, explorar y descubrir. }\end{array}$ \\
\hline & Deseo de aprender & Deseo de conocer, mejorar, manejar nuevas habilidades. \\
\hline & $\begin{array}{l}\text { Apertura a la } \\
\text { experiencia }\end{array}$ & $\begin{array}{l}\text { Examinar bajo diferentes puntos de vista, tener en cuenta } \\
\text { todas las características de la situación antes de opinar sobre } \\
\text { ellas o tomar una decisión y ser capaz de cambiar de opinión } \\
\text { ante la evidencia. }\end{array}$ \\
\hline
\end{tabular}

Perspectiva

Ser capaz de proporcionar un sabio consejo a los demás; tener maneras de ver el mundo que hace que tenga sentido para uno mismo y para los otros.

\begin{tabular}{|c|c|c|}
\hline Valor & Valor/Valentía & $\begin{array}{l}\text { No echarse atrás por el miedo, los cambios, las dificultades } \\
\text { o el dolor; defender lo que es justo a pesar de la oposición } \\
\text { y actuar de acuerdo a las convicciones aunque sean } \\
\text { impopulares. }\end{array}$ \\
\hline
\end{tabular}

Perseverancia

Acabar lo que uno empieza; persistir en una acción a pesar de los obstáculos; concentrarse en lo que se hace y dedicar esfuerzo a conseguir objetivos. 


\begin{tabular}{|c|c|c|}
\hline & Integridad & $\begin{array}{l}\text { Decir la verdad, vivir de forma genuina y auténtica y ser } \\
\text { responsable de los propios sentimientos y acciones. }\end{array}$ \\
\hline & Vitalidad & $\begin{array}{l}\text { Vivir de forma apasionada y con energía, como si la vida } \\
\text { fuera una aventura. }\end{array}$ \\
\hline \multirow[t]{3}{*}{ Amor /humildad } & Intimidad & $\begin{array}{l}\text { Amar y dejarse amar, valorar las relaciones íntimas y } \\
\text { profundas con los demás, ser cercano a la gente. }\end{array}$ \\
\hline & Amabilidad & Hacer favores a los otros, ayudarles y cuidarles. \\
\hline & Inteligencia social & $\begin{array}{l}\text { Conocimiento de uno mismo y de los demás, conocer cómo } \\
\text { actuar en las situaciones sociales y cómo hacer que los otros } \\
\text { se sientan bien. }\end{array}$ \\
\hline \multirow[t]{3}{*}{ Justicia } & Ciudadanía & $\begin{array}{l}\text { Trabajar bien en equipo, ser fiel y apoyar los objetivos } \\
\text { comunes. }\end{array}$ \\
\hline & Justicia & $\begin{array}{l}\text { Tratar a todo el mundo con los mismos criterios de justicia; } \\
\text { no permitir que los sentimientos personales interfieran en } \\
\text { las actuaciones con los demás. }\end{array}$ \\
\hline & Liderazgo & $\begin{array}{l}\text { Animar al grupo para conseguir juntos los objetivos } \\
\text { propuestos, manteniendo al mismo tiempo buenas relaciones } \\
\text { en él. }\end{array}$ \\
\hline \multirow[t]{4}{*}{ Templanza } & $\begin{array}{l}\text { Capacidad de } \\
\text { perdonar }\end{array}$ & $\begin{array}{l}\text { Olvidar lo que otros nos han hecho y herido; darles una } \\
\text { segunda oportunidad; no ser vengativo. }\end{array}$ \\
\hline & Humildad & $\begin{array}{l}\text { No intentar ser el centro de atención, no tratarse a uno mismo } \\
\text { como más especial de lo que se es. }\end{array}$ \\
\hline & Prudencia & $\begin{array}{l}\text { Ser cauto a la hora de tomar decisiones; no asumir riesgos } \\
\text { innecesarios. }\end{array}$ \\
\hline & Autorregulación & $\begin{array}{l}\text { Regular lo que uno siente o piensa; ser disciplinado; controlar } \\
\text { los apetitos y necesidades. }\end{array}$ \\
\hline \multirow[t]{5}{*}{ Trascendencia } & $\begin{array}{l}\text { Apreciación de } \\
\text { la belleza }\end{array}$ & $\begin{array}{l}\text { Percibir y apreciar la belleza o excelencia de cualquier faceta } \\
\text { de la vida. }\end{array}$ \\
\hline & Gratitud & $\begin{array}{l}\text { Ser consciente de las cosas buenas que suceden y dar gracias } \\
\text { por ellas, dedicando tiempo a expresarlo. }\end{array}$ \\
\hline & $\begin{array}{l}\text { Optimismo } \\
\text { Esperanza }\end{array}$ & $\begin{array}{l}\text { Esperar lo mejor del futuro y trabajar para lograrlo; confiar } \\
\text { en que el futuro depare cosas buenas. }\end{array}$ \\
\hline & Humor & $\begin{array}{l}\text { Tendencia a reír y sonreír; hacer reír a otras personas; ver } \\
\text { el lado cómico de lo que sucede. }\end{array}$ \\
\hline & Espiritualidad & $\begin{array}{l}\text { Poseer creencias fuertes y coherentes sobre la razón y } \\
\text { significado trascendente del universo; saber cuál es su lugar } \\
\text { en el orden universal; apoyarse en estas creencias para actuar } \\
\text { y sentirse reconfortado. }\end{array}$ \\
\hline
\end{tabular}

El Values in Action Inventory of Strenghts (VIAIS, Seligman y Peterson, 2000 tomado de Peterson y Seligman, 2004) evalúa las 24 fortalezas en adultos. Los autores han puesto disponible en Internet esta versión del cuestionario (http:// www.authentichappiness.org) a través de la cual han conseguido obtener resultados de una amplia y diversa muestra (más de 175 naciones), incluida una versión 
española disponible en la página web: http://www.psicologiapositiva.org.

El Values in Action Inventory of Strenghts for Youth (VIAY, Peterson, Seligman, Dahlsgaard y Park, 2003 tomado de Peterson y Seligman, 2004) es el cuestionario adaptado para adolescentes entre 10 y 17 años. Recientemente hemos realizado una adaptación al castellano de este cuestionario y aplicado a adolescentes entre 12 y 18 años que completaron además otras medidas de bienestar y psicopatología. Los resultados apuntan relaciones positivas significativas entre las diferentes fortalezas y satisfacción con la vida, autoestima y afecto positivo y relaciones negativas con variables psicopatológicas como la depresión o el estrés (Giménez, 2010). Cabe destacar además que las fortalezas que se repiten sistemáticamente asociadas a bienestar (Gratitud, Intimidad, Vitalidad y Optimismo), son las mismas que se asocian de forma negativa con problemas psicopatológicos y el papel mediacional de las fortalezas entre el clima familiar percibido y la satisfacción global con la vida.

\section{FORTALEZAS PSICOLÓGICAS EN ADOLESCENTES}

A pesar de la importancia que se da al proceso de construcción de la identidad y a la formación de valores en los adolescentes, no son demasiados los estudios que abordan el desarrollo de las fortalezas personales (Park, 2004; Huebner, Gilman y Furlong, 2009). En este sentido, nos ha parecido oportuno hacer una revisión de cada una de las fortalezas psicológicas recogiendo los datos más significativos obtenidos en diferentes investigaciones, haciendo especial hincapié en los estudios con población adolescente española.

\section{Fortalezas de la sabiduría y el conocimiento}

Desde el punto de vista estricto de aprendizaje, las fortalezas de este grupo (Creatividad, Curiosidad, Apertura a la Experiencia, Deseo de aprender y Perspectiva) podrían considerarse las más importantes. Durante la adolescencia, los chicos y las chicas se encuentran en un período esencial en su formación; frente a esto, los datos apuntan que es el momento en el que los resultados académicos descienden, aumenta el abandono escolar y disminuye el interés hacia las actividades escolares (Samdal, Nutbeam, Wold, y Kannas 1998; Redy, Rhodes, y Mulhall, 2003). En este momento, el adolescente parece prestar menos atención a las asignaturas que se imparten en la clase, pero también es posible que surjan nuevos ámbitos de interés (normalmente ajenos a la escuela) en los que deposite su motivación y deseos de aprender.

Si bien no cabe duda de la relevancia de este grupo de fortalezas, nos encontramos con pocos estudios que aborden su estudio (Giménez, 2010). Sabemos, por ejemplo, que los adolescentes son capaces de idear alternativas y de imaginar nuevas soluciones, de enfrentarse a los problemas de una forma creativa (Fernández, Villaoslada y Funes, 2002). También que la Sabiduría (o Perspectiva), fortaleza que se ha situado tradicionalmente en el último estadio de la vida (Erikson, 
1968), tiene sus periodos iniciales de desarrollo (adquisición del conocimiento asociado a sabiduría) entre los 15 y los 25 años (Staudinger y Pasupathi, 2003) y que estaría asociado a los cambios cognitivos característicos de esta etapa (desarrollo del pensamiento moral y capacidad de adoptar diferentes perspectivas).

En relación a la Curiosidad y la Apertura a la experiencia, ser curioso/a y estar abierto a variedad de pensamientos, perspectivas e ideas facilita el aprendizaje y un mejor rendimiento académico (Wavo, 2004; Kashdan y Yuen, 2007), un efecto que no puede ser atribuido a la propia capacidad intelectual (Reiss y Reiss, 2004). Naturalmente ésta, como otras capacidades, no puede entenderse sin el contexto y el carácter (positivo) de las instituciones que rodean al individuo. De hecho, diversos estudios han demostrado la relación entre el desarrollo de la curiosidad y ciertas características del ambiente escolar (Skinner, Wellborn y Connell, 1990), especialmente del apoyo percibido del profesor (Black y Deci, 2000).

En cuanto al Deseo de aprender, se ha encontrado que presenta una relación positiva con la motivación intrínseca (Anderman y Young, 1994; Miller, Behrens, Green y Newman, 1993), deseo de superación, interés en el propio desarrollo y valor del aprendizaje (Zimmerman, Bandura y MartínezPons, 1992).

A partir de algunos indicadores, diversos estudios han encontrado diferencias en función del sexo con algunos conceptos asociados a este grupo de fortalezas. Por ejemplo, parece ser que los chicos presentan en mayor medida una orientación motivacional extrínseca (Cerezo y Casanova, 2004) y las chicas una mayor motivación intrínseca (Meece y Holt, 1993), aunque no siempre se encuentran estas diferencias (Ryan y Pintrich, 1997). Por otro lado, los chicos parecen hacer uso de menos estrategias de aprendizaje significativo (Cerezo y Casanova, 2004) al estar más centrados en ofrecer una imagen positiva de sí mismos. Y por último, se ha encontrado que existe una estrecha relación entre el conocimiento asociado a la sabiduría y el rendimiento académico, si bien está modulado por el sexo: en las chicas existe una relación positiva y significativa, mientras que en los chicos no se da dicha relación (González Leandro y Pelechano, 2004).

Estos resultados sugieren que hay ciertos elementos contextuales que pueden ayudar a explicar estas diferencias (p.ej., el tipo de disciplina, la imagen asociada al rol femenino y masculino o las expectativas que se depositan en los chicos y en las chicas).

\section{Fortalezas de coraje}

Desgraciadamente existen muy pocos estudios sobre este importante grupo de fortalezas, tal y como son propuestas en el modelo de Peterson y Seligman (2004), por lo que optamos por revisar estudios que evalúan conceptos con los que pudieran tener cierta semejanza.

Algunos autores han enfatizado el papel del valor en el desarrollo del adolescente ya que éste tiene que enfrentarse a situaciones adversas en su día a día que están ligadas al proceso de construcción de su propia identidad (Way, 1998). 
Atendiendo a indicadores indirectos de la perseverancia como pudieran ser la tasa de respuesta en una tarea (desde una aproximación conductual la persistencia puede estimarse a partir de esta medida), o el abandono escolar, se encuentra que las jóvenes españolas permanecen más tiempo en el sistema educativo y se titulan en mayor proporción en la educación superior que sus compañeros (según datos de la Organización para la Cooperación y el Desarrollo Económico, 2006). Hay un número mucho mayor de varones que dejan de estudiar entre los 15 y los 17 años (Martín y Velarde, 2000). Ahora bien, sería necesario conocer la persistencia en otros ámbitos o tareas para poder establecer conclusiones consistentes sobre posibles diferencias de género y profundizar en los beneficios de esta fortaleza.

Como indicador de Vitalidad, podemos utilizar, también a modo de indicador indirecto, uno de sus antónimos: la fatiga, una de las quejas más habituales entre los adolescentes. Wolbeek, Van Doornen, Kavelaars y Heijnen (2006) han encontrado una elevada prevalencia de la fatiga intensa en los adolescentes y diferencias significativas entre chicos $(6,5 \%)$ y chicas $(20,5 \%)$. Tradicionalmente la fatiga es atribuida a los cambios hormonales propios de la etapa, así como al aumento de las demandas sociales y educativas a las que están expuestos los adolescentes. Ahora bien, y esto es una tarea que debe ser sometida a investigaciones futuras, expresar o manifestar fatiga no tiene porqué excluir experimentar otras emociones relacionadas con la vitalidad y el entusiasmo.

\section{Fortalezas interpersonales}

Dentro de este grupo se han propuesto el Amor/intimidad, la Amabilidad y la Inteligencia Social. Se trata de las fortalezas que permiten al ser humano relacionarse con los demás y desarrollar los vínculos afectivos necesarios para sentirse libre, apoyado y seguro. No podemos olvidar que la relaciones con otras personas se consideran uno de los pilares básicos de satisfacción con la vida (Deci y Ryan, 2000).

El adolescente asume en esta etapa de su vida la tarea de ser más autónomo respecto a sus padres y buscar otras figuras con las que vincularse: los iguales (p.ej.: Overbeek, Vollebergh, Engels y Meeus, 2003; Hazan, 2004). Ahora bien, pese a lo que tradicionalmente se piensa, esto no significa que los padres dejen de ser referentes en la vida de los adolescentes, simplemente empiezan a necesitar de sus progenitores cosas que no demandaban en su infancia (Hernangómez, Vázquez y Hervás, 2009).

Sentirse afectivamente seguro permite a los adolescentes y adultos ser más capaces de enfrentarse al estrés diario y desarrollar estrategias para establecer relaciones sociales más satisfactorias (Hazan, 2004). Las relaciones seguras establecidas con los padres se relacionan positivamente con éxito personal, social y académico en los escolares (Rice, Fitzgerald, Whaley y Gibbs, 1995) y se asocian a un menor consumo de tabaco y alcohol (Martínez y Robles, 2001). Por otro lado, relaciones inseguras con los padres pueden contribuir al desarrollo de 
síntomas depresivos (Sund y Wichstrom, 2002), ansiedad y evitación de explorar el entorno y relacionarse con los demás (Lopez y Brennan, 2000) y relaciones con iguales conflictivos y conductas de riesgo o antisociales (Serrano, Godás, Rodríguez y Mirón, 1996).

Los adolescentes españoles expresan una actitud positiva hacia sus familias, a las que perciben como un espacio facilitador y protector, y hacia sus iguales, encontrándose un nivel más alto de sociabilidad en las chicas que en los chicos (Serrano et al., 1996).

Dentro de la fortaleza Amabilidad podrían estudiarse variables como el altruismo, la conducta prosocial o la empatía. Durante la adolescencia, parecen incrementarse las actitudes altruistas gracias al desarrollo de nuevas aptitudes cognitivas que favorecen la empatía o capacidad para ponerse en el lugar del otro como tomar conciencia de que las experiencias vitales transcienden la situación inmediata (Etxebarria, 2001).

Los adolescentes prosociales tienden a presentar una tendencia empática hacia los demás (Calvo, González y Martorell, 2001; Mestre, Samper y Frías, 2002), puntuaciones más altas en autoconcepto y autoestima (Calvo, González y Martorell., 2001) y una mayor autorregulación cognitiva y emocional (Tur, Mestre y Barrio, 2004).

Como es sabido, el Modelo de Cinco Factores (McCrae y Costa, 1995) incluye la Amabilidad para el estudio de la personalidad. Se ha encontrado que la baja amabilidad y la baja responsabilidad correlacionan de forma significativa con el consumo de alcohol tanto en los chicos como en las chicas adolescentes (Mezquita et al., 2006), que los jóvenes violentos presentan niveles más bajos de responsabilidad y amabilidad (Tur, Mestre y Barrio, 2004) y que la baja amabilidad es predictor de agresividad (especialmente la física) en chicos, pero no en chicas (Carrasco y Barrio, 2007). Diferentes estudios han encontrado puntuaciones significativamente más altas en amabilidad en las adolescentes (Ortet et al., 2007).

En cuanto a la Inteligencia Social, existen pocos estudios que analicen esta variable. En población adolescente española, se ha encontrado una relación negativa entre inteligencia social y síntomas obsesivos/compulsivos, sensibilidad intepersonal (sentirse inferior, hipersensible a la opinión de los demás), depresión, ansiedad, hostilidad, ansiedad fóbica, ideación paranoide, psicoticismo y melancolía (Garaigordobil, 2006)

\section{Fortalezas de justicia}

En las fortalezas de justicia, los autores incluyen Ciudadanía, Equidad y Liderazgo. Dentro de la Ciudadanía encontramos la responsabilidad social, la lealtad y el trabajo en equipo (aunque existen diferencias notables entre los conceptos) que tienen en común el sentimiento de obligación con el bien común por encima de los propios intereses (Flanagan, 2004). En cuanto a correlaciones con otras variables, se sabe que los adolescentes que participan al menos en una actividad social tienen una imagen más positiva de los demás (Flanagan, Gill y 
Gallay, 2005). Frente a esto, en las últimas encuestas con jóvenes encontramos que el asociacionismo no es algo demasiado frecuente (Elzo, 2006).

En relación al trabajo en equipo, se pueden extraer algunas conclusiones analizando los resultados que evalúan cooperación. En un estudio con 322 adolescentes españoles de entre 14 y 17 años, Garaigordobil (2006) encuentra que los adolescentes que actúan cooperativamente en actividades de trabajo o tiempo libre y que disfrutan compartiendo acciones que potencian las relaciones sociales, tienen pocos síntomas psicopatológicos (p.ej., menos síntomas obsesivocompulsivos, de depresión, de ansiedad, de hostilidad y de ansiedad fóbica).

Sobre la Equidad, sabemos que a lo largo de la adolescencia, el chico o la chica puede ir adquiriendo las capacidades cognitivas necesarias para lograr una moral autónoma y posconvencional, es decir, un modo de razonamiento más universal y fundamentado. Por ello, durante este período, a medida que avanza la edad se consiguen mayores puntuaciones en estadios de mayor nivel de razonamiento moral (p. ej.: PérezDelgado, Frías y Pons, 1994).

En la actualidad, el liderazgo adolescente es considerado una herramienta esencial no sólo como habilidad que posibilita el desarrollo integral del potencial del chico o de la chica que la posee, sino que se convierte en una forma de promover y consolidar cambios entre adolescentes y jóvenes. Un ejemplo de ello son los denominados programas de ayuda entre iguales que consisten en la creación y formación de un colectivo del alumnado que sea capaz de escuchar y acompañar en sus necesidades a otros alumnos (o adultos de la comunidad educativa), basándose en las dificultades que experimentan y proporcionando herramientas para resolverlas desde la negociación y el respeto mutuo (Fernández. Villaoslada y Funes, 2002). Por desgracia, a pesar de su gran importancia potencial, nuevamente nos encontramos con pocos estudios que hayan analizado en profundidad esta fortaleza.

\section{Fortalezas de templanza}

Dentro de las fortalezas de templanza se encuentran el Perdón, la Humildad, la Prudencia y la Autorregulación.

Las relaciones con los demás cobran una gran importancia durante la adolescencia. Cabe esperar que la intensidad de las emociones, el alto grado de implicación en las relaciones con otros y las dificultades asociadas al proceso de construcción de la propia identidad, provoque que existan más conflictos con los otros que en otras etapas de la vida. En este sentido, la capacidad de perdonar, por ejemplo, se convierte en una fortaleza esencial para el intercambio social del adolescente.

En un estudio con adolescentes italianos se encontró que la calidad de las relaciones paternas predecía atribuciones benignas hacia las conductas negativas de los padres. Es decir, los adolescentes perdonaban las conductas de sus padres si existía una buena relación con ellos, lo cual se asociaba a una menor probabilidad de conflicto entre ambos (Paleari, Regalia y Fincham, 2003). Otros 
estudios han encontrado cierta relación entre la comprensión del perdón por parte de adolescentes coreanos y la conducta de perdonar en situaciones reales (Park y Enright, 1997).

En relación a la Humildad, podemos encontrar algunos indicadores de esta fortaleza en los estudios que han evaluado habilidades sociales. Por ejemplo, el cuestionario de Matson para la Evaluación de Habilidades Sociales con Adolescentes (MESSY; Matson, Rotatori y Helsel, 1983) incluye un factor denominado Vanidad/arrogancia o Sobrevaloración que, por oposición, ofrece información sobre la humildad en este periodo de la vida. En un estudio con población adolescente, la Vanidad/arrogancia correlacionaba de forma significativa y positiva con conducta asocial, agresividad, extraversión y psicoticismo, y de forma negativa con asertividad y sumisión; además, las chicas puntuaron más bajo en esta escala que los chicos (Méndez, Hidalgo e Inglés, 2002). En un estudio llevado por Garaigordobil (2006), también con población adolescente, se encontraron correlaciones significativas entre el factor Sobrevaloración del MESSY y diferente sintomatología psicológica como la ansiedad, la hostilidad, la fobia, la depresión, la ideación paranoide y el psicoticismo evaluados a través del SCL90R, lo que podría sugerir una interesante relación entre humildad y una mayor salud mental y probablemente una mayor madurez y autoaceptación.

La Modestia está incluida como una faceta del factor Amabilidad evaluado a través del NEOPIR (Costa y McCrae, 1999), un cuestionario de personalidad que evalúa el Modelo de Cinco Factores en adolescentes. Estudios con adolescentes españoles han encontrado que dicha faceta correlaciona de manera significativa y negativa con afecto negativo y con conducta antisocial (Romero, Luengo, GómezFraguela y Sobral, 2002).

Para evaluar la Prudencia, nuevamente debemos recurrir a conceptos con los que consideramos que existe cierta similitud, en este caso, el de responsabilidad. En adolescentes españoles se ha encontrado que la Responsabilidad (evaluada a través del NEOPIR, Costa y McCrae, 1999) correlaciona de forma negativa con Neuroticismo y positivamente con Amabilidad. Además, las chicas obtienen mayores puntuaciones en Responsabilidad que los chicos (Ortet et al., 2007). En cuanto a otras variables, se ha encontrado que Responsabilidad se asocia tanto con un alto Afecto Positivo como con un bajo Afecto Negativo, con conducta antisocial y con fracaso escolar (Romero et al., 2002).

Por último, la mayor parte de la investigación sobre el papel de la autoregulación se ha centrado en explorar los efectos del maltrato en ésta (p.ej. Cicchetti, Ackerman e Izard, 1995) o en niños propensos a emocionalidad negativa o con dificultades sociales (p.ej.: Fabes et al., 2002) y apenas existe soporte científico del papel de la auto-regulación como protección (Buckley y Saarni, 2009). En educación se ha centrado en un aspecto específico del concepto: el aprendizaje autoregulado, es decir, en las estrategias cognitivas, afectivomotivacionales y comportamentales que el estudiante utiliza para conseguir objetivos académicos (Valle et al., 2003). En este sentido, la autoregulación permite al estudiante prepararse para el éxito inmediato desarrollando buenos hábitos de estudio y a plan- 
tearse objetivos a largo plazo de cara a conseguir lo que planea para su futuro.

\section{Fortalezas transcendentales}

En este último grupo se incluye la Apreciación de la Belleza, la Gratitud, la Esperanza, el Humor y la Espiritualidad.

El cuestionario de personalidad NEOPIR recoge, dentro del factor Apertura a la experiencia, la faceta de Estética. Atendiendo a estudios en población española que han utilizado este instrumento, se ha encontrado una correlación positiva y significativa tanto con el afecto positivo como con el negativo (Romero et al., 2002) y mayores puntuaciones en las chicas que en los chicos (Ortet et al., 2007).

A pesar de los beneficios de la gratitud (p.ej., McCulloug, Emmons y Tsang, 2002), son pocos los estudios que se centran en niños y adolescentes (Bono y Froh, 2009). Uno de los primeros acercamientos es el realizado por Froh, Yurkewicz y Kashdan (2008) que encontraron una relación positiva entre el nivel de gratitud y una variedad de indicadores positivos como el afecto positivo, satisfacción con la vida, optimismo, apoyo social y conducta prosocial, y una relación negativa con síntomas físicos. También se ha encontrado una relación importante entre gratitud y satisfacción con la experiencia escolar (Froh, Sefick, y Emmons, 2008).

En muestras de adolescentes españoles, se encuentra alguna referencia que estudia el agradecimiento en el contexto de las habilidades sociales. En este sentido, los adolescentes manifiestan que las conductas de dar las gracias (ejemplo de ítem: “'Tienes dificultad para dar las gracias a tu amigo/a cuando te ayuda en tus tareas escolares?”) son las consideradas más fáciles de realizar frente a otras situaciones interpersonales como, por ejemplo, "Relaciones con el otro sexo" o "Hablar en público" (Inglés, Méndez e Hidalgo, 2000).

La investigación sobre Esperanza y Optimismo se ha centrado en su importancia como factor protector ante la adversidad y el estrés y la promoción del ajuste psicológico (Seligman et al., 1995; Russo y Boman, 2007; Cassidy, 2000; Yarckeski, Mahon y Yarcheski, 2004; Chang y Sanna, 2003). Un pensamiento esperanzador está asociado, en la infancia, con percepción de competencia y autoestima (Marques, PaisRibeiro y Lopez, 2007), y negativamente con síntomas de depresión (Kwon, 2000). En población española, encontramos que, en la encuesta realizada por Javaloy (2007), a partir de la pregunta: "Pensando en el futuro, ¿crees que tu vida mejorará un poco, continuará igual, empeorará un poco o empeorará mucho?", se encontró que el $86,3 \%$ de los jóvenes españoles pensaba que su vida mejorará en el futuro. De modo que una visión positiva sobre el futuro parece ser la tónica general de los jóvenes de nuestro país, aunque los cambios en la situación económica general puede que modifiquen esta perspectiva optimista global,

En relación al Humor, los primeros estudios con niños y adolescentes están proporcionando información valiosa sobre las funciones cognitivas, sociales, defensivas y fisiológicas del mismo. Tal y como resumen a partir de la literatura 
al respecto Erickson y Feldstein (2007), los niños con "buen sentido el humor" tienden a ser más asertivos y competentes en contextos sociales y académicos. Estos autores sugieren que el sentido del humor puede jugar un papel importante como estrategia de afrontamiento y defensiva en los adolescentes. En un estudio realizado con una pequeña muestra $(\mathrm{N}=113)$ encuentran que las formas benignas del humor aparecen asociadas con estrategias de afrontamiento de aproximación (frente a las de evitación) y a estilos defensivos maduros (frente a estilos que niegan o distorsionan el conflicto), con menos síntomas depresivos y con un mejor ajuste personal.

En cuanto a la Espiritualidad, sabemos que en la adolescencia y juventud, se ha encontrado que la religiosidad está asociada con la evitación de actividades antisociales (Maton y Wells, 1995). Ahora bien, la espiritualidad debe entenderse como algo más amplio, e incluso diferente, que la identificación con una opción religiosa. El sentido de la vida, por ejemplo, es una valoración a tener en cuenta dentro de esta fortaleza. En el estudio coordinado por Javaloy (2007), la mayoría de los jóvenes entrevistados estaba de acuerdo con la afirmación: "Mi vida está llena de sentido" (sólo el 11,4\% decía estar muy o bastante en desacuerdo). Los autores, además, constataron relaciones positivas entre dar sentido a la vida y el nivel percibido de control sobre el mundo, y la felicidad percibida.

\section{CONCLUSIONES}

La adolescencia es un período de la vida en el que los procesos de construcción de identidad, el desarrollo de nuevas formas de pensamiento, incluyendo la capacidad de razonamiento moral y el fortalecimiento de relaciones sociales, se convierten en una oportunidad irrepetible para la consolidación de valores y fortalezas humanas. Padres, educadores y sociedad en general no deben perder de vista las posibilidades asociadas a esta etapa de la vida. Una visión centrada en las carencias, el conflicto o la vulnerabilidad, resulta insuficiente para capturar la complejidad del comportamiento humano. Incluso en áreas de conflicto (Ortega, 2010), una visión complementaria de las fortalezas existentes, puede ser un elemento importante para completar el diagnóstico y realizar intervenciones más eficaces.

La revisión realizada en el presente trabajo nos ha permitido constatar la falta de un marco común desde el que abordar el estudio de las cualidades positivas de los adolescentes y la escasez de estudios que evalúan algunas de estas cualidades de forma aislada. Paradójicamente desde los diferentes ámbitos educativos se habla de la importancia de la perseverancia, el respeto hacia los demás o el autocontrol, pero son pocos los estudios que se centran en estos conceptos.

Hablar de valores y de fortalezas humanas no puede quedar reducido a una serie de buenas intenciones o sujeto, únicamente, a reflexiones morales o filosóficas. Cada vez es más necesario que estos conceptos se incluyan en un estudio objetivo que no entre tanto a valorar las virtudes más importantes en sí, sino aquellas que mejor contribuyen al desarrollo pleno de la persona, incluyendo en 
éste la vinculación con los demás: un pensamiento y modo de vida que actúe con responsabilidad hacia uno mismo y los otros.

Tal y como propone Seligman (2003), para entender la experiencia humana desde un punto de vista positivo es necesario tener en cuenta tres elementos: un análisis de las experiencias positivas (que incluye estudiar el bienestar psicológico y la satisfacción con la vida, entre otros), favorecer rasgos individuales (formación del carácter y fortalezas) y analizar las características de las instituciones positivas (familia, escuela y sociedad en general) que faciliten el desarrollo de los dos primeros. No cabe duda de que reconocer y analizar mejor las fortalezas psicológicas será una vía importante para favorecer las condiciones de una buen infancia y crear sistemas educativos positivos (Layard y Dunn, 2009).

En este sentido, nos parecen fundamentales instrumentos como el VIAY que evalúan los aspectos y cualidades más positivas del individuo. Desde el punto de vista práctico, este tipo de medidas permiten evaluar los programas de prevención e intervención que desde diferentes organismos e instituciones se promueven y que tienen como objetivo el desarrollo positivo de cualquier grupo.

Conocer las fortalezas humanas que se asocian con satisfacción y con patología nos proporciona pistas muy importantes a la hora de establecer los objetivos de dichos programas. Park (2004) propone que aquellas fortalezas más relacionadas con satisfacción con la vida deberían ser las primeras en incluirse en los programas diseñados para el desarrollo positivo de los jóvenes; en este caso, las mismas fortalezas contribuirían a los dos objetivos (mejorar el bienestar y reducir el riesgo de padecer un problema psicológico). El optimismo, la vitalidad, la autoestima y relaciones positivas con los demás podrían estar en la base de estas intervenciones.

Asimismo, el estudio y la evaluación de las fortalezas puede ser muy relevante en los contextos de orientación vocacional, en donde en ocasiones prima el empleo de test de intereses con poco calado psicológico. En este campo, la adecuada comprensión de las fortalezas innatas del adolescente podría ser una alternativa de gran riqueza y un instrumento para conseguir que el asentamiento de la identidad y el desarrollo vital y vocacional de la persona se construya de forma sólida y coherente.

A partir de la revisión realizada, constatamos una vez más la importancia de la familia y del contexto escolar para el desarrollo y el fortalecimiento de cualidades positivas en los más jóvenes. En este sentido, merece la pena prestar atención a las diferencias encontradas en función del sexo. Las diferentes investigaciones apuntan el papel que desempeñan los diferentes roles sociales en el desarrollo de todas estas variables (Cerezo y Casanova, 2004; Bono y Froh, 2009).

En este sentido, es fundamental analizar los componentes que están configurando una educación diferenciada entre los dos sexos y estudiar en profundidad sus efectos, no tanto para apostar por un único tipo de educación, pero sí para descubrir qué componentes permiten a las personas, independientemente de su sexo, recibir la educación que le favorece el máximo desarrollo de sus potencialidades. 
Nos referimos por tanto a una intervención educativa amplia, no al desarrollo único de programas extracurriculares. Los responsables de la planificación y programación de los contenidos, procedimientos y actitudes pueden partir de una conceptualización positiva del adolescente, en términos de potencialidades y no sólo de déficits, lo que permitirá sin duda un desarrollo más pleno.

En resumen, a pesar de las dificultades de las sociedades modernas, los riesgos asociados a la adolescencia y los cambios intrínsecos de esta etapa de la vida, los resultados encontrados dibujan una situación bastante esperanzadora en relación a esta población y apuntan importantes implicaciones en el desarrollo de actuaciones y programas específicos para el pleno aprovechamiento de las fortalezas así como su potencial desarrollo.

\section{REFERENCIAS}

Allen, J. P. y Land, D. (1999). Attachment in Adolescence. En J. Cassidy y P. Shaver (Eds.), Handbook of attachment (pp. 319-335). New York: The Guilford Press.

Anderman, E.M. y Young, A.J. (1994). Motivation and strategy use in science: Individual differences and classroom effects. Journal of Research in Science Teaching, 31, 811-831.

Arguedas, I. y Jiménez, F. (2007). Factores que promueven la permanencia de estudiantes en la educación secundaria. Revista Electrónica "Actualidades Investigativas en Educación”, 7, 1-36.

Black, A.E. y Deci, E.L. (2000). The effects of instructors'autonomy support and students'autonomous motivation on learning organic chemistry: a selfdetermination theory perspective. Science Education, 84, 740-756.

Bono, G., y Froh, J. J. (2009). Gratitude in school: Benefits to students and schools. En R. Gilman, E. S. Huebner, y M. Furlong (Eds.)., Handbook of positive psychology in the schools: Promoting wellness in children and youth (pp.77-88). Hillsdale, NJ: Lawrence Erlbaum.

Calvo, A. J., González, R. y Martorell, M. C. (2001). Variables relacionadas con la conducta prosocial en la infancia y adolescencia: personalidad, autoconcepto y género. Infancia y Aprendizaje, 24, 95-111.

Cassidy, T. (2000). Social background, achievement motivation, optimism and health: a longitudinal study. Counselling Psychology Quarterly, 13, 399-412.

Carrasco, M. A. y Barrio, $\mathrm{M}^{\mathrm{a}}$ V. (2007). El modelo de los cinco grandes como predictor de la conducta agresiva en población infantojuvenil. Revista de Psicopatología y Psicología Clínica, 12, 23-32.

Cerezo, M.T y Casanova, P.F. (2004). Diferencias de género en la motivación académica de los alumnos de Educación Secundaria Obligatoria. Revista electrónica de investigación psicoeducativa, 2, 97-112.

Chang, E.C. y Sanna, L.J. (2003). Optimism, accumulated life stress, and psychological and physical adjustment: Is it always adaptative to expect the best? Journal of Social \& Clinical Psychology, 22, 97-115. 
Cicchetti, D., Ackerman, B. e Izard, C. (1995). Emotions and emotion regulation in developmental psychopatology. Development and Psychopatology, 7, 1-10.

Cook, W. L. (2000). Understanding attachment security in family context. Journal of Personality and Social Psychology, 78, 285-294.

Corral, S., Pamos, A., Pereña, J. y Seisdedos, N. (2002). TPT. Test de Personalidad de TEA. Madrid: TEA.

Costa, P.T. y McCrae, R.R. (1999). Inventario de personalidad NEO revisado (NEO-PI-R) e Inventario NEO reducido de cinco factores (NEO-FFI). Manual profesional. Madrid. TEA.

Deci, E.L. y Ryan, R.M. (2000). The "what" and "why" of goal pursuits: human needs and the selfdetermination of behaviour. Psychological Inquiry, 11, 227-68.

Elzo, J. (2006). Valores e identidades en los jóvenes. En González Blasco (Dir.), Jóvenes Españoles 2005. Madrid: Fundación Santamaría. SM.

Etxebarria, I. (2001). Desarrollo del altruismo y la agresión. En López, F., Extebarria, I., Fuentes, M.J. y Ortiz M.J. (Coords.). Desarrollo afectivo y social (pp.211-230). Madrid, Pirámide.

Erikson, E. (1968). Identidad, juventud y crisis. Buenos Aires: Editorial Paidós.

Erickson, S.J. y Feldstein, S.W. (2007). Adolescent Humor and its relationship Coing, Defense Strategies, Psychological Distress, and Well Being. Child Psychiatry and Human Development, 37, 255-271.

Fabes, R., Hanish, L., Martin, C.L. y Eisenberg, N. (2002). Young children's negative emotionality and social isolation: a latent growth curve analysis. MerrillPalmer Quarterly, 48, 284-307.

Fernández, I., Villaoslada, E. y Funes, S. (2002). Conflicto en el centro escolar. El modelo de "alumno ayudante" como estrategia de intervención educativa. Madrid: Catarata.

Fernández-Ballesteros, R. (2002): Light and dark in the psychology of human strengths: The example of psychogerontology. En L.G. Aspinwal y U.M. Staudinger (Eds.), A Psychology of Human Strength: Perspectives on an Emerging Field. Washington DC: APA.

Flanagan, C.A. (2004). Citizenship. Social Responsibility, Loyalty, Teamwork. En C. Peterson y M.E.P. Seligman (Eds.). Character strengths and virtues: a handbook and classification (pp. 369389). Washington, DC: American Psychological Association and Oxford University Press.

Flanagan, C.A., Gill, S., y Gallay, L.S. (2005). Social participation and social trust in adolescence: The importance of heterogeneous encounters. En A. Omoto, (Ed.), Processes of community change and social action (pp. 149166). Erlbaum.

Froh, J.J., Sefick, W.J. y Emmons, R.A. (2008). Counting blessings in early adolescents: an experimental study of gratitude and subjective wellbeing. Journal of School Psychology, 46, 213-233.

Froh, J.J., Yurkewicz, C. y Kashdan, T.B. (2008). Gratitude and subjective wellbeing in early adolescence: Examining gender differences. Journal of Ado- 
lescence, DOI:10.1016/j.adolescence.2008.06.006

Garaigordobil, M. (2006). Psychopathological Symptoms, Social Skills, and Personality Traits: a Study with Adolescents. The Spanish Journal of Psychology, 9, 182-192.

Giménez, M. (2010) La medida de las fortalezas psicológicas en adolescentes (VIAY): relación con clima familiar, psicopatología y bienestar psicológico. Tesis doctoral no publicada, Facultad de Psicología, Universidad Complutense, Madrid, España.

González Leandro, P. y Pelechano, V. (2004). Sabiduría contemporánea, motivación y rendimiento académico en adolescentes. Análisis y Modificación de conducta, 30, 963-976.

Hazan, C. (2004). Love. En C. Peterson y M.E.P. Seligman (Eds.), Character strengths and virtues: a handbook and classification (pp. 303-324). Washington, DC: American Psychological Association and Oxford University Press.

Hernangómez, L., Vázquez, C. y Hervás, G. (2009) El paisaje emocional a lo largo de la vida. La ciencia del bienestar psicológico. En C. Vázquez y G. Hervás (Eds.), La ciencia del bienestar: Fundamentos de una Psicología Positiva (pp.143-179). Madrid: Alianza Editorial.

Huebner, E.S., Gilman, R. y Furlong, M.J. (2009). A conceptual model for research in positive psychology in children and youth. En R. Gilman, E. S. Huebner, y M. Furlong (Eds.)., Handbook of positive psychology in the schools: Promoting wellness in children and youth (pp.3-8). Hillsdale, NJ: Lawrence Erlbaum.

Inglés, C.J., Méndez, F.X. e Hidalgo, M.D. (2000). Cuestionario de Evaluación de Dificultades Interpersonales en la Adolescencia. Psicothema, 12, 390-398.

Javaloy, F. (Coord.). (2007). Bienestar y felicidad de la juventud española. Madrid: INJUVE.

Kashdan, T.B. y Yuen, M. (2007). Wether highly curious students thrive academically depends on perceptions about the school learning environment: a study of Hong Kong adolescents. Motivation and Emotion, 31, 260-270.

Layard, R. y Dunn, J. (2009). A good childhood. Searching for values in a competitive age. Londres: Penguin.

Lopez, F. G., y Brennan, K. A. (2000). Dynamic processes underlying adult attachment organization: Toward an attachment theoretical perspective on the healthy and effective self. Journal of Counseling Psychology, 47, 283-301.

Marques, S.S., Pais-Ribeiro, J.L. y Lopez, S.J. (2007) Hope in relation to life satisfaction, mental-health, and self-worth in students. Póster presentado en el $10^{\circ}$ Congreso Europeo de Psicología, Praga, República Checa.

Martín, S. y Velarde, O. (2001). Informe Juventud en España 2000. Madrid: INJUVE. Martínez, J.M. y Robles, L. (2001). Variables de protección ante el consumo de alcohol y tabaco en la adolescencia. Psicothema, 13, 222-228.

Maton, K. y Wells, E. (1995). Religion as a community resource for wellbeing: Prevention, healing, and empowerment pathways. Journal of Social Issues, 51, 177-193. 
Matson, J.L., Rotatori, A.F. y Helsel, W.J. (1983). Development of a rating scale to measure social skills in children: The Matson Evaluation of Social Skills with Youngsters (MESSY). Behaviour Research and Therapy, 21, 335-340.

McCrae, R.R. y Costa, P.T. (1995). Trait explanations in personality psychology. European Journal of Personality, 9, 231-252.

McCulloug, M.E., Emmons, R.A. y Tsang, J. (2002). The grateful disposition: a conceptual and empirical topography Journal of Personality and Social Psychology, 82, 112-127.

Meece, J.L. y Holt, K. (1993). A pattern análisis of students' achievement goals. Journal of Educational Psychology, 85, 582-590.

Méndez, F.X., Hidalgo, M.D. e Inglés, C.J. (2002). The Matson Evaluation of Social Skills with Youngsters. Psychometric Properties of the Spanish Translation in the Adolescente Population. European Journal of Psychological Assessment, 18, 30-42.

Mestre, V., Samper, P. y Frías, M. D. (2002). Procesos cognitivos y emocionales predictores de la conducta prosocial y agresiva: La empatía como factor modulador. Psicothema, 14, 227-232.

Mezquita, L., Maestre, E., Mestre, H., Viñas, M., Moya, J. y Ortet, G. (2006). Relación entre personalidad y consumo de alcohol en adolescentes españoles y escoceses. Jornades de Foment de la Investigació. Universitat Jaume I. Castellón: España. Consultado el 28 de marzo de 2009 en: http://www.uji.es/ bin/publ/edicions/jfi11/2.pdf

Miller, B.R., Behrens, J.T., Green, B.A. y Newman, D. (1993). Goals and perceived ability: Impact on student valuing, self-regulation and persistence. Contemporary Educational Psychology, 18, 2-14.

Ortega, R. (2010). (Coord.). Agresividad injustificada, bullying y violencia escolar. Madrid: Alianza.

Ortet, G., Ibañez, M.I, Ruipérez, M.A., Villa, H., Moya, J. y Escrivá, P. (2007). Adaptación para adolescentes de la versión española del NEO PIR (JS NEO). Psicothema, 19, 263-268.

Overbeek, G., Vollebergh, W., Engels, C. y Meeus, W. (2003). Parental attachment and romantic relationships. Associations with emotional disturbance during late adolescence. Journal of Counseling Psychology, 50, 28-39.

Paleari, F.G., Regalia, C. y Fincham, F.D. (2003). Adolescents' willingness to forgive their parents: an empirical model. Parenting, 3, 155-174.

Park, N. (2004). The role of subjective wellbeing in positive youth development. The Annals of the American Academy of Political and Social Science, 591, 2539.

Park, Y.O. y Enright, R.D. (1997). The development of forgiveness in the context of adolescent friendship conflict in Korea. Journal of Adolescence, 20, 393402.

PérezDelgado, E., Frías, D. y Pons, G. (1994). El cuestionario de problemas morales (DIT). de J. Rest (1979) y su estructura. En E. PérezDelgado y M.J. Soler (Eds.), El Cuestionario de Problemas Sociomorales (DIT) de J. Rest y 
su uso informatizado (pp.2550). Valencia: Albatros Ediciones.

Peterson, C. y Seligman, M.E.P. (Eds.) (2004). Character strengths and virtues: a handbook and classification. Washington, DC: American Psychological Association and Oxford University Press.

Reddy, R., Rhodes, J. y Mulhall, P. (2003). The influence of teacher support on student adjustment in the middle school years: A latent growth curve study, Development and Psychopathology, 15, 119-138.

Reiss, S. y Reiss, M.M. (2004). Curiosity and mental retardation: beyond IQ. Mental Retardation, 42, 77-81.

Rice, K. G., Fitzgerald, D. P., Whaley, T. J., y Gibbs, C. L. (1995). Cross-sectional and longitudinal examination of attachment, separationindividuation, and college student development. Journal of Counseling and Development, 73, 463-474.

Romero, E., Luengo, M.A., GómezFraguela, J.A. y Sobral, J. (2002). La estructura de los ragos de personalidad en adolescentes: el modelo de Cinco Factores y los Cinco Alternativos. Psicothema, 14, 134-143.

Russo, R. y Boman, P. (2007). Primary school teachers'ability to recognise resilience in their students. The Australian Educational Researcher, 34, 17-31.

Ryan, A.M. y Pintrich, P.R. (1997). Should I ask for help? The role of motivation and attitudes in adolescents' helpseeking in math class. Journal of Educational Psychology, 89, 329-341.

Samdal, O., Nutbeam, D., Wold, B. y Kannas, L. (1998). Achieving health and educational goals through schools: a study of the importance of the school climate and students' satisfaction with school. Health Education Research, 13, 383-397.

Seligman, M.E.P. (2003). La auténtica felicidad. Barcelona, Ediciones Vergara.

Seligman, M.E.P., Reivich, K., Jaycox, L. y Gillham, J. (1995). The optimistic child. New Cork Houghton Mifflin.

Serrano, G., Godás, A., Rodríguez, D. y Mirón, L. (1996). Perfil psicosocial de los adolescentes españoles. Psicothema, 8, 25-44.

Sheldon, K. M., y Elliot, A. J. (1999). Goal striving, needsatisfaction, and longitudinal wellbeing: The SelfConcordance Model. Journal of Personality and Social Psychology, 76, 482-497.

Skinner, E.A., Wellborn, J.G. y Connell, J.P. (1990). What it takes to do well in school and wether I've got it: The role of perceived control in children's engagement and school achievement. Journal of Educational Psychology, 82, 22-32.

Snyder, C.R., Hoza, B., Pelham, W.E., Rapoff, M., Ware, L, Danovsky, M., Highberger, L., Ribinstein, H. y Stahl, K.J. (1997). The development and validation of the Childrens' Hope Scale. Journal of Pediatric Psychology, 22, 399-421.

Staudinger, .U.M. y Pasupathi, M. (2003). Correlates of WisdomRelated Performance in Adolescence and Adulthood: AgeGraded Differences in "Paths" Toward Desirable Development. Journal of Research on Adolescence, 13, 
239-268.

Sund, A.M. y Wichstrom, L. (2002). Insecure Attachment as a Risk Factor for Future Depressive Symptoms in Early Adolescence. Journal of the American Academy of Child and Adolescent Psychiatry, 41, 1478-1485.

Tur, A. M., Mestre, M. V. y Barrio, M. V. (2004). Factores moduladores de la conducta agresiva y prosocial. El efecto de los hábitos de crianza en la conducta del adolescente. Ansiedad y Estrés, 10, 75-88.

Valle, A., González-Cabanach, R., Núñez, J.C., González-Pienda, J.A., Rodríguez, S. y Piñeiro, I. (2003). Cognitive, motivational, and volitional dimensions of learning: An empirical test of a hypothetical model. Research in Higher Education, 44, 557-580.

Vázquez, C. (2009). La ciencia del bienestar. En C. Vázquez y G. Hervás (Eds.), El estudio cientifico del bienestar: Fundamentos de una Psicología Positiva (pp. 13-46). Madrid: Alianza Editorial.

Vázquez, C. y Hervás, G. (2009) (Eds.), La ciencia del bienestar: Fundamentos de una Psicología Positiva. Madrid: Alianza Editorial.

Wavo, E.Y.T. (2004). Honesty, cooperation and curiosity achievement of some schools on Nanjing (China). IFE Psychologia: An International Journal, 12, 178-188.

Way, N. (1998). Everyday courage: the lives and stories of urban teenagers. New York: New York University Press.

Wolbeek, M., Van Doomen, L.J.P., Kavelaars, A. y Heijnen, C.J. (2006). Severe Fatigue in Adolescents: A Common Phenomenon? Pediatrics, 117, 10781086. Consultado el 28 de marzo de 2009 en: http://www.pediatrics.org/cgi/ content/full/117/6/e1078

Yarcheski, T.J., Mahon, N.E. y Yarcheski, A. (2004). Depression, optimism, and positive health practices in young adolescents. Pscychological Reports, 95, 932-934.

Zimmerman, B.J., Bandura, A. y MartínezPons, M. (1992). Self-motivation for academic attainment: the role of selfefficacy beliefs and personal goal setting. American Educational Research Journal, 29, 663-676. 